\title{
Reconciling the Diversity and Uniformity of Galactic Rotation Curves with Self-Interacting Dark Matter
}

\author{
Tao Ren, ${ }^{1}$ Anna Kwa, ${ }^{2}$ Manoj Kaplinghat, ${ }^{2, *}$ and Hai-Bo $\mathrm{Yu}^{1, \dagger}$ \\ ${ }^{1}$ Department of Physics and Astronomy, University of California, Riverside, California 92521, USA \\ ${ }^{2}$ Department of Physics and Astronomy, University of California, Irvine, California 92697, USA
}

(Received 23 October 2018; revised manuscript received 25 April 2019; published 7 August 2019)

Galactic rotation curves exhibit diverse behavior in the inner regions while obeying an organizing principle; i.e., they can be approximately described by a radial acceleration relation or the modified Newtonian dynamics phenomenology. We analyze the rotation curve data from the SPARC sample and explicitly demonstrate that both the diversity and uniformity are naturally reproduced in a hierarchical structure formation model with the addition of dark matter self-interactions. The required concentrations of the dark matter halos are fully consistent with the concentration-mass relation predicted by the Planck cosmological model. The inferred stellar mass-to-light $(3.6 \mu \mathrm{m})$ ratios scatter around $0.5 M_{\odot} / L_{\odot}$, as expected from population synthesis models, leading to a tight radial acceleration relation and a baryonic Tully-Fisher relation. The inferred stellar-halo mass relation is consistent with the expectations from abundance matching. These results provide compelling arguments in favor of the idea that the inner halos of galaxies are thermalized due to dark matter self-interactions.

DOI: 10.1103/PhysRevX.9.031020

Subject Areas: Astrophysics, Cosmology

\section{INTRODUCTION}

Galactic rotation curves of spiral galaxies show a variety of behaviors in the inner parts, even across systems with similar halo and stellar masses, which lacks a self-consistent explanation in the standard cold dark matter (CDM) model [1-13]. Along with this diversity, a long-standing observation is that many rotation curves can be understood in terms of modified Newtonian dynamics (MOND) phenomenology [14,15] (see Ref. [16] for a review); i.e., there exists a characteristic gravitational acceleration scale, $g_{\dagger} \approx 10^{-10} \mathrm{~m} / \mathrm{s}^{2} \sim c H_{0} / 7$, with $H_{0}$ being the present Hubble expansion rate, below which the observed acceleration can be approximated as $\sqrt{g_{\dagger} g_{\text {bar }}}$, with $g_{\text {bar }}$ being the baryonic acceleration (a.k.a. Milgrom's law). More recently, McGaugh et al. [17] analyzed the Spitzer Photometry and Accurate Rotation Curves (SPARC) data set [18] and showed that there is a tight relation between the total gravitational acceleration at any radius and the acceleration contributed by the baryons, assuming a

\footnotetext{
* Corresponding author.

mkapling@uci.edu

Corresponding author. haiboyu@ucr.edu

Published by the American Physical Society under the terms of the Creative Commons Attribution 4.0 International license. Further distribution of this work must maintain attribution to the author(s) and the published article's title, journal citation, and DOI.
}

constant stellar mass-to-light ratio $\Upsilon_{\star \text {,disk }}=0.5 M_{\odot} / L_{\odot}$ and $\Upsilon_{\star \text {,bulge }}=0.7 M_{\odot} / L_{\odot}$ in the 3.6- $\mu \mathrm{m}$ band. The scatter in this radial acceleration relation (RAR) is around $0.1 \mathrm{dex}$, and the tightness of this relation has been interpreted as a signature of MOND [19].

It has long been argued that the acceleration scale (including the $\mathrm{cH}_{0}$ dependence) can emerge from hierarchical structure formation predicted in CDM [20,21]. Recent hydrodynamical simulations of galaxy formation with CDM have clearly shown that a RAR emerges [22-24]. However, these simulated galaxies do not represent the full range of diversity in the SPARC data set, and they cannot yet explain the rotation curves of low- and high-surface-brightness galaxies simultaneously.

In this paper, we show that self-interacting dark matter (SIDM) provides a unified way to understand the diverse rotation curves of spiral galaxies while reproducing the RAR with a small scatter. We analyze the SPARC data set based on the SIDM halo model proposed in Refs. [25,26] and demonstrate three key observations leading to this result.

(i) For cross section per unit mass $\sigma / \mathrm{m} \sim 1 \mathrm{~cm}^{2} / \mathrm{g}$, dark matter self-interactions thermalize the inner regions at distances less than about $10 \%$ of the virial radius of galactic halos, while the outer regions remain unchanged. Thus, SIDM inherits essential features of the $\Lambda \mathrm{CDM}$ hierarchical structure formation model such as the halo concentration-mass relation, which sets the characteristic acceleration scale of halos. 
(ii) In the inner halo, thermalization ties dark matter and baryon distributions together $[25,27,28]$, and the SIDM halo can naturally accommodate the diverse range of "cored" and "cusped" central density profiles, depending on how the baryons are distributed. Combined with the scatter in the concentration-mass relation, this provides the diversity required to explain the rotation curves $[26,29,30]$.

(iii) For the same $\sigma / \mathrm{m}$ that addresses the diversity problem, the baryon content of the galaxies and the mass model of their host halos also lead to the RAR with a scatter as small as the one in Ref. [17]. In our SIDM fits, the inferred stellar $\Upsilon_{\star \text {,disk }}$ values for individual galaxies have a distribution peaked toward $0.5 M_{\odot} / L_{\odot}$, as expected from stellar population synthesis models [31].

The rest of the paper is organized as follows. In Sec. II, we present the SIDM fits to 135 galaxies from the SPARC sample, which exemplify the full range of the diversity. In Sec. III, we show the radial acceleration relation and the distribution of the stellar mass-to-light ratios from our SIDM fits, compared to the MOND fits. In Sec. IV, we discuss the host halo properties and the origin of the acceleration scale. In Sec. V, we show the predicted stellar-halo mass relation and the baryonic Tully-Fisher relation (BTFR). We comment on future directions and conclude in Sec. VI. In the Appendix, we provide detailed information about the model and the fitting procedure. In the Supplemental Material [32], we present SIDM and MOND fits to 135 individual galaxies from the SPARC sample and additional results that support the main text, including model fits to simulated halos.

\section{THE DIVERSITY OF GALACTIC ROTATION CURVES}

We select 135 out of 175 galaxies in the full SPARC sample based on the criteria that they must have a recorded value for the flat part of the rotation curve, $V_{\mathrm{f}}$. In our sample, 87, 42, and 6 galaxies have quality flags 1,2 , and 3 , respectively. It spans a wide range of galaxy masses and inner shapes of rotation curves with $V_{\mathrm{f}}$ ranging from $20 \mathrm{~km} / \mathrm{s}$ to $300 \mathrm{~km} / \mathrm{s}$. In fitting to the data, we utilize the analytical SIDM halo model [26,29], where we assume the dark matter distribution in the inner halo follows the isothermal density profile,

$$
\rho_{\text {iso }}(R, z)=\rho_{0} \exp \left(\left[\Phi_{\text {tot }}(0,0)-\Phi_{\text {tot }}(R, z)\right] / \sigma_{\mathrm{v} 0}^{2}\right),
$$

where $\rho_{0}$ is the central dark matter density, $\sigma_{\mathrm{v} 0}$ is the one-dimensional dark matter velocity dispersion, $\Phi_{\text {tot }}(R, z)$ is the total gravitational potential, and $R, z$ are cylindrical coordinates aligned with the stellar disk.
We match this isothermal profile to a Navarro-FrenkWhite (NFW) form [33,34] at $r_{1}$, where a dark matter particle has scattered $\mathcal{O}(1)$ times over the age of the galaxy, assuming continuity in both the density and the enclosed mass at $r_{1}$. In this way, the isothermal parameters $\left(\rho_{0}, \sigma_{\mathrm{v} 0}\right)$ directly map onto the NFW parameters $\left(r_{s}, \rho_{s}\right)$ or $\left(r_{\max }, V_{\max }\right)$. This model provides an approximate way to calculate the SIDM distribution in a halo if its CDM counterpart is known, and vice versa. It correctly predicts the halo central density and its scalings with the outer halo properties, stellar profiles, and cross section, as confirmed in both isolated and cosmological N-body simulations with and without baryons; see, e.g., Refs. [26,28,30,35,36]. See the Appendix and the Supplemental Material [32] for a detailed description of the model and additional comparisons between model predictions and cosmological simulations.

We adopt two independent but complementary approaches to perform the analysis. In the controlled sampling (CS) approach, we demand that the host halos follow the concentration-mass relation within a $2 \sigma$ range predicted in cosmological simulations [37]. We model the stellar distribution as an axisymmetric thin disk as in Ref. [29], which directly enters into the calculation of the density profile of SIDM through the gravitational potential $\Phi(R, z)$. In the CS fits, we start with the outer NFW halo and find the SIDM density profile that matches its mass and density at $r_{1}$. In the second approach, we use the Markov chain Monte Carlo (MCMC) sampling (MS) to explore the full likelihood. To save computational time, we assume spherical symmetry by spreading the mass within the disk at radius $R$ into a sphere of the same radius $[25,28]$. The rotation curves generated from two approaches agree well, and the differences in the fits are small (see Supplemental Material [32]). For our main results, we show inferences from both of the approaches.

In Fig. 1, we show the SIDM fits to the diverse rotation curves from the controlled sampling with $\sigma / \mathrm{m}=3 \mathrm{~cm}^{2} / \mathrm{g}$. In each panel, galaxies are selected to have similar flat rotation velocities at their outermost data points. The rise up to $V_{\mathrm{f}}$ within their central regions displays a wide variety of slopes, and the SIDM halo model provides equally good fits to the shallow and steeply rising rotation curves. The fits for the other galaxies in the sample are as good as those in Fig. 1 (see Supplemental Material [32]).

The success of the SIDM halo model stems from a combination of the following effects. First, SIDM thermalization ties the baryon and dark matter distributions together. For low-surface-brightness galaxies, thermalization leads to a shallow density core and a circular velocity profile that rises mildly with radius [38-44]. While, for high-surfacebrightness galaxies, the core shrinks in response to the deeper baryonic potential, and the central SIDM density increases accordingly $[25,28,30,36]$. The galaxies in our 

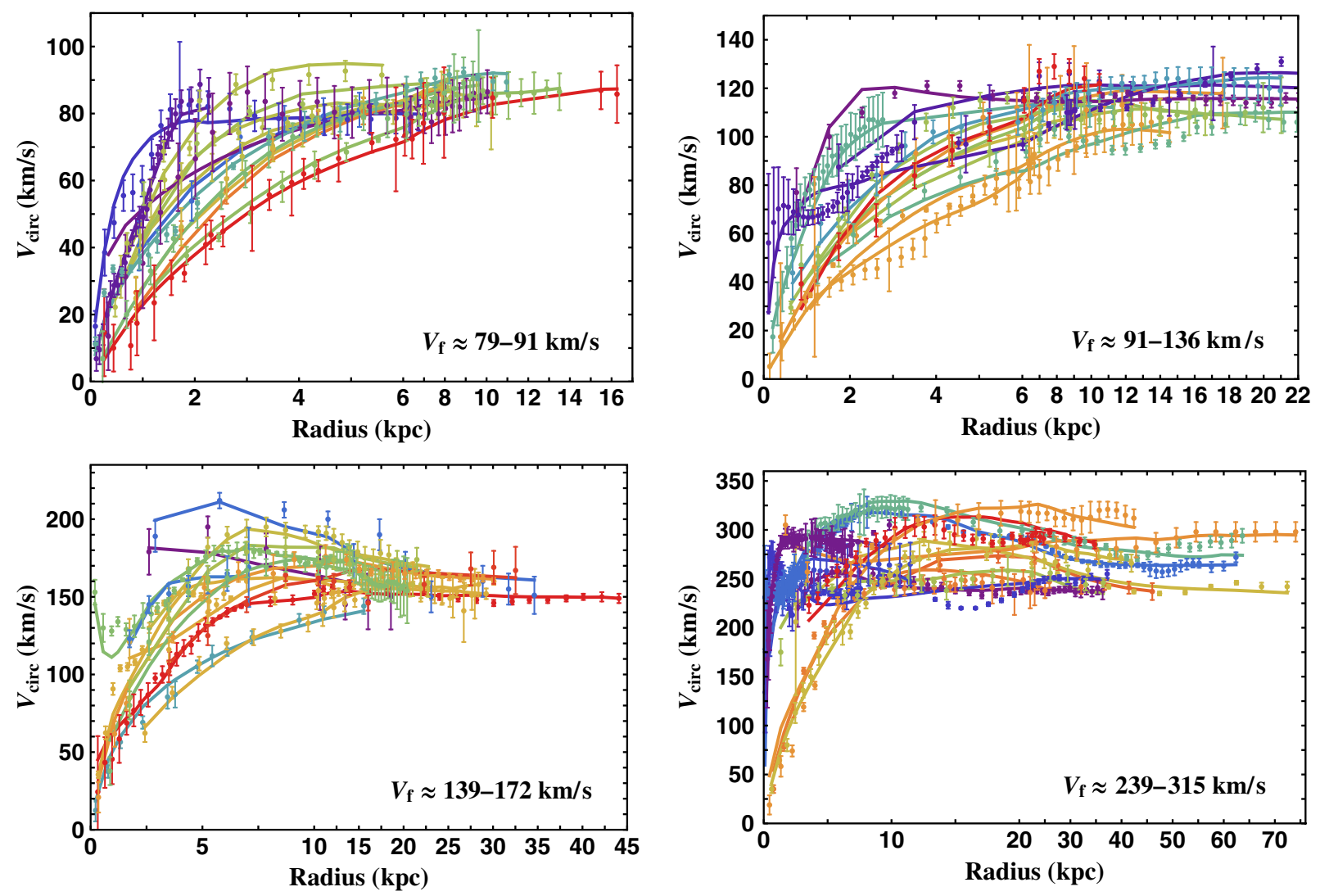

FIG. 1. SIDM fits (solid) to the diverse rotation curves across a range of spiral galaxy masses, where we take $\sigma / \mathrm{m}=3 \mathrm{~cm} / \mathrm{g}$. The data points with error bars are from the SPARC data set [18]. Each panel contains 14 galactic rotation curves that are selected to have similar flat rotation velocities at their furthest radial data points, and the corresponding $V_{\mathrm{f}}$ bins are 79-91, 91-126, 139-172, and 239-315 km/s, spanning the mass range of the galaxies considered in this work. The galaxies are colored according to their relative surface brightness in each panel from low (red) to high (violet).

sample have a variety of central surface brightnesses, resulting in diverse central dark matter densities. Second, scatter in the cosmological halo concentration-mass relation leads to scatter in the characteristic SIDM core density and radius, which is reflected in the rotation curves [26]. Reference [29] fitted 30 galaxies and illustrated the importance of these effects in explaining the diverse rotation curves. In this work, we fit a larger sample of galaxies and demonstrate that the observed galaxies are fully consistent with the SIDM predictions.

We have assumed a constant cross section to fit the SPARC sample because it is hard to pin down the cross section for individual galaxies. For low-surface-brightness galaxies with a large core, a large cross section, such as $\sigma / \mathrm{m}=3 \mathrm{~cm}^{2} / \mathrm{g}$, is preferred [29]. However, since the central SIDM density varies mildly with the cross section in the range $1-10 \mathrm{~cm}^{2} / \mathrm{g}[45,46]$, a feature that is well captured in our analytical model [26], an even larger cross section may work as well. For high-surface-brightness galaxies, to which most of the galaxies with high $V_{\mathrm{f}}$ belong, the fits are insensitive to the cross section because of the degeneracy between $\sigma / \mathrm{m}$ and $\Upsilon_{\star}$ [29]. The effect in the SIDM fits induced by varying $\sigma / \mathrm{m}$ can be compensated by a minor change in the stellar mass-to-light ratio, and many of these systems are actually compatible with a NFW profile. The cross section may have a mild velocity dependence over the sample, as implied by the constraint from galaxy clusters $[26,47,48]$, but it is impossible to extract it from the SPARC data set given the reasons discussed above. In this work, we present the results for fixed $\sigma / \mathrm{m}=3 \mathrm{~cm}^{2} / \mathrm{g}$, and they remain the same qualitatively for other values larger than about $1 \mathrm{~cm}^{2} / \mathrm{g}$ on galaxy scales.

An important consequence of the large cross section is that the SIDM profile is driven quickly to be isothermal in the inner regions. This implies that the resultant SIDM fits will not depend sensitively on the formation history of individual galaxies [29] but on the final stellar and gas distributions [25]. This has been explicitly confirmed in recent hydrodynamical SIDM simulations [35] and those with idealized disk growth [28]. Furthermore, in our fits $r_{1}$ is close to $r_{s}$, which is well outside the stellar disk or budge in the galaxies. It is unlikely that a viable baryonic feedback process could change the halo mass profile significantly at that far distance. Thus, our analytical model takes into account the realistic baryon distribution for individual 

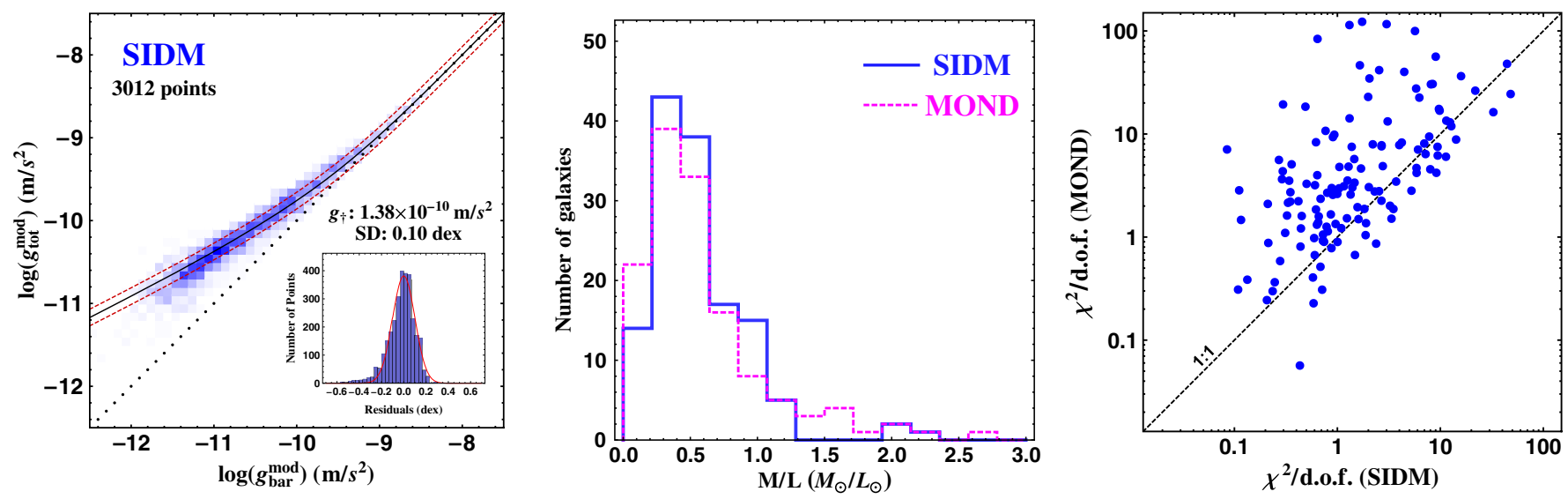

FIG. 2. Left panel: The radial acceleration relation from the SIDM fits, where $g_{\mathrm{tot}}^{\mathrm{mod}}$ and $g_{\mathrm{bar}}^{\bmod }$ are inferred from the $\sigma / \mathrm{m}=3 \mathrm{~cm}{ }^{2} / \mathrm{g}$ fits. The black solid line is the best fit to Eq. (2); the two red dashed curves correspond to the $1 \sigma$ deviation from this fit. The black dotted line is the one-to-one reference line. Insets: Corresponding histograms of residuals after subtracting the fit function with the best-fitting scale parameter $g_{\dagger}=1.38 \times 10^{-10} \mathrm{~m} / \mathrm{s}^{2}$, together with the Gaussian fits to the residuals, which have $1 \sigma$ widths of 0.10 dex. Middle panel: Inferred $\Upsilon_{\star \text {,disk }}$ distributions for the SIDM and MOND fits. Right panel: Distribution of $\chi^{2} /$ d.o.f. values for individual galaxies from the SIDM and MOND fits.

galaxies and encodes this effect on the SIDM halo profile through the matching procedure.

\section{THE RADIAL ACCELERATION RELATION IN SIDM}

In the RAR described in Ref. [17], the gravitational acceleration $g_{\text {tot }}$ at radius $r$ is found to be related to the acceleration $g_{\mathrm{bar}}$ at the same radius. This relation can be fit to a functional form with a single parameter $g_{\dagger}$ :

$$
g_{\mathrm{tot}}(r)=g_{\mathrm{bar}}(r)\left(1-e^{-\sqrt{g_{\mathrm{bar}}(r) / g_{\dagger}}}\right)^{-1}
$$

Their best-fit value of $g_{\dagger}=1.2 \times 10^{-10} \mathrm{~m} / \mathrm{s}^{2}$ is the oftquoted MOND acceleration scale.

In the left panel of Fig. 2, we show the inferred total and baryonic acceleration values from the controlled sampling, where $g_{\mathrm{tot}}^{\bmod }$ and $g_{\mathrm{bar}}^{\bmod }$ are calculated from the SIDM fits, using the halo parameters and the best-fit $\Upsilon_{\star}$ values for each galaxy. The intensity of color in Fig. 2 (left panel) reflects the density of points. After fitting the data with the empirical relation given in Eq. (2), we find that the best-fit value of $g_{\dagger}$ is $1.38 \times 10^{-10} \mathrm{~cm}^{2} / \mathrm{g}$, and the resulting dispersion in the residuals is 0.10 dex. Figure 2 (middle panel) shows $\Upsilon_{\star \text {,disk }}$ distribution from the SIDM fits (solid line). It is peaked toward $\Upsilon_{\star \text {,disk }}=0.5 M_{\odot} / L_{\odot}$, in $\operatorname{good}$ agreement with predictions from stellar population synthesis models [31]. This result is remarkable because no priors based on the stellar population synthesis models were used. We have also reproduced the analysis in Ref. [17], with $\Upsilon_{\star \text {,disk }}$ and $\Upsilon_{\star \text {,bulge }}$ fixed to $0.5 M_{\odot} / L_{\odot}$ and $0.7 M_{\odot} / L_{\odot}$, respectively. For this fixed $\Upsilon_{\star}$ case, we obtain $g_{\dagger}=1.19 \times 10^{-10} \mathrm{~m} / \mathrm{s}^{2}$ and dispersion $0.12 \mathrm{dex}$, both in agreement with previous work [17].

For a more detailed comparison, we also fit the sample of 135 SPARC galaxies using the MOND relation in Eq. (2), where we fix $g_{\dagger}=1.2 \times 10^{-10} \mathrm{~m} / \mathrm{s}^{2}$ but vary $\Upsilon_{\star \text {,disk }}$ and $\Upsilon_{\star \text {,bulge }}$ in the range of $0.1<\Upsilon_{\star} /\left(M_{\odot} / L_{\odot}\right)<10$ (same as MCMC SIDM fits) using MCMC sampling (see also Ref. [49]). The results look similar if we set $g_{\dagger}$ to $1.0 \times$ $10^{-10}$ or $1.4 \times 10^{-10} \mathrm{~m} / \mathrm{s}^{2}$. The middle panel of Fig. 2 shows the $\Upsilon_{\star \text {,disk }}$ distribution from the MOND fits (dotted line), which closely tracks the one from the SIDM fits. The right panel shows the distribution of minimum $\chi^{2} /$ d.o.f. values for individual galaxies from the SIDM and MOND fits. The SIDM model provides a better fit than MOND for most of the galaxies $(\sim 77 \%)$ while maintaining a tight RAR. In fact, $72 \%$ (45\%) of them have $\chi^{2} /$ d.o.f. $\leq 3(1)$ in the controlled SIDM fits, and those with a large $\chi^{2} /$ d.o.f. value have either tiny errors or wiggles in the observed rotation curves that cannot be reproduced by MOND either. We have also compared our fits with the MOND fits in Li et al. [19]. The major difference is that they marginalized over both the distance and inclination uncertainties, while we did not. The MOND fits in Ref. [19] are slightly better than our MOND fits due to the two additional variables (distance and inclination), but still only about $20 \%$ of the galaxies are fit with $\chi^{2} /$ d.o.f. $\leq 1$. For comparison, we have checked that over $60 \%$ of the MCMC SIDM fits have $\chi^{2} /$ d.o.f. $\leq 1$.

We emphasize that the diversity in the inner rotation curves is also reflected in the $g_{\text {tot }}-g_{\text {bar }}$ plane, as explicitly demonstrated in the Supplemental Material [32], where we show the $g_{\text {tot }}$ vs $g_{\text {bar }}$ plot but now split the sample into two sets: radii outside and inside $2 R_{\mathrm{d}}$, with $R_{\mathrm{d}}$ being the scale 

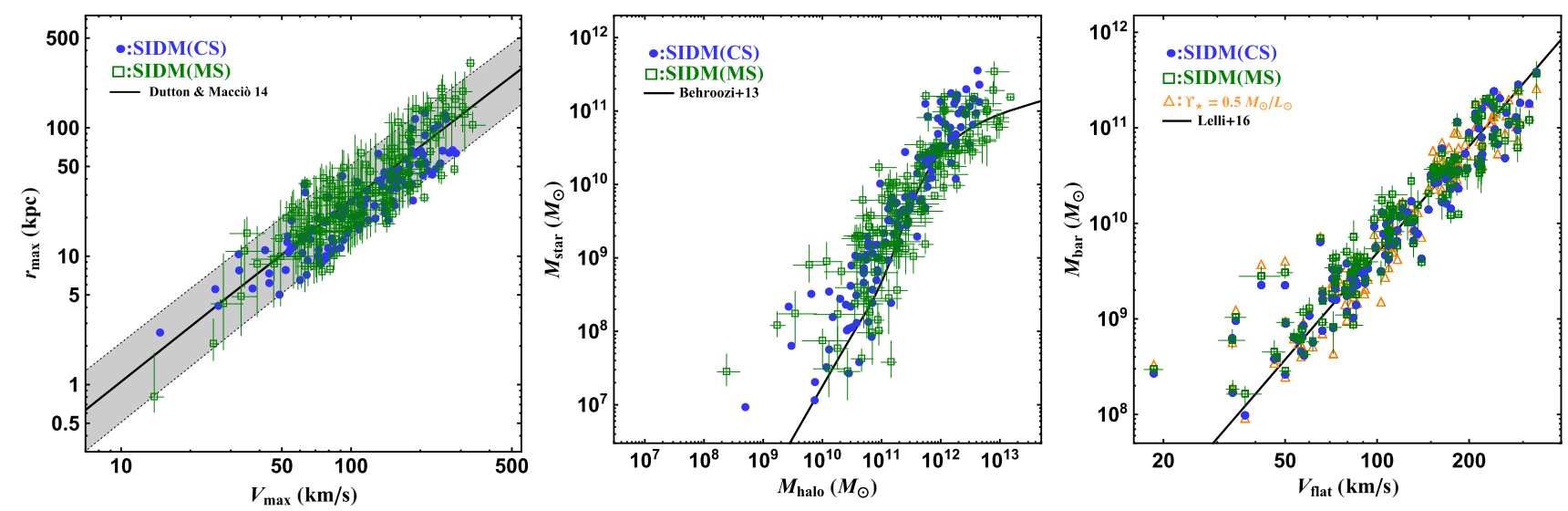

FIG. 3. Left panel: $r_{\max }-V_{\max }$ distributions of the host halos in the SIDM fits with controlled (circles) and MCMC (squares) samplings. We also show the mean relation (black solid line) and $2 \sigma$ scatter (gray shaded area) predicted in cosmological CDM simulations [37]. Middle panel: Halo virial mass vs galaxy stellar mass from the SIDM fits. The black solid line corresponds to the abundance matching inference from Ref. [58]. Right panel: Total baryonic mass vs flat circular velocity for the 135 galaxies, where $M_{\text {bar }}$ is inferred from our SIDM fits (circles and squares). For comparison, we also show the case (triangles) when $\Upsilon_{\star \text {,disk }}$ and $\Upsilon_{\star \text {,bulge }}$ are fixed to $0.5 M_{\odot} / L_{\odot}$ [54]. The black solid line is the mean baryonic Tully-Fisher relation from Ref. [54], derived from 118 SPARC galaxies with $\Upsilon_{\star \text {,disk }}=\Upsilon_{\star \text {,bulge }}=0.5 M_{\odot} / L_{\odot}$, at which the scatter is minimized.

radius of the stellar disk. The scatter is relatively large for radii less than $2 R_{\mathrm{d}}$, and this is due to the different shapes in the inner rotation curves and not just the result of random errors (see also Ref. [50]). On the other hand, there is a clear ordered behavior of $g_{\mathrm{tot}}$ vs $g_{\mathrm{bar}}$ curves for radii $>2 R_{\text {disk }}$, which is a reflection of the BTFR: the tight correlation between the flat circular velocity $V_{\mathrm{f}}$ and the total baryonic mass $M_{\text {bar }}$ for spiral galaxies [51]. In this regime, $g_{\mathrm{tot}} \approx \sqrt{g_{\dagger} g_{\mathrm{bar}}}$, where $g_{\mathrm{tot}} \approx V_{\mathrm{f}}^{2} / r$ and $g_{\text {bar }} \approx G M_{\text {bar }} / r^{2}$; hence, we have $V_{\mathrm{f}}^{4} /\left(G M_{\text {bar }}\right) \approx g_{\dagger}$. This is the success of MOND; i.e., if one assumes $M_{\mathrm{bar}} \propto V_{\mathrm{f}}^{4}$, then the normalization of the BTFR also predicts the rotation curve, which in many cases is a good fit to the observed one. Many studies find $M_{\mathrm{bar}} \propto V_{\mathrm{f}}^{s}$ with $3<s<4$ [52-54], as we also show in Sec. V; $s=4$ is not forced upon us by the data, but it is not ruled out either. However, the MOND relation [Eq. (2)] cannot explain the full range of the diversity in the inner rotation curves, while the success of SIDM is deeply rooted in hierarchical structure formation, as we discuss in the next section.

\section{CONCENTRATION-MASS RELATION AND ORIGIN OF THE CHARACTERISTIC ACCELERATION SCALE}

We have demonstrated that SIDM explains both the diversity and the tight RAR exhibited in the rotation curves, as dark matter self-interactions thermalize the inner halo in the presence of the baryonic potential. Here, we show that the host halos in the SIDM fits are consistent with predictions in the hierarchical structure formation model; see, e.g., Refs. [37,55,56]. Since the outer halo $\left(r \gtrsim r_{1}\right)$ remains unchanged for $\sigma / \mathrm{m}=3 \mathrm{~cm}^{2} / \mathrm{g}$, we parametrize a
SIDM halo using the concentration and mass or, equivalently, the maximal circular velocity $\left(V_{\max }\right)$ and the associated radius $\left(r_{\max }\right)$ of its CDM counterpart. Ideally, one would measure these halo parameters directly from the kinematics data and compare them with simulations. Unfortunately, most rotation curves do not have the radial extent needed to sufficiently constrain them. In this work, we impose the cosmological concentration-mass relation [37] as a prior similar to Ref. [57] and examine the consistency between its consequences and observations.

In Fig. 3 (left panel), we show the $r_{\max }-V_{\max }$ distributions from our controlled (circles) and MCMC (squares) samplings. For the former, we intend to seek the best SIDM fits to the rotation curves following the mean relation (solid line) from simulations. For the sample we consider, $97 \%$ of galaxies can be fitted within the $2 \sigma$ band (gray shaded area), calculated from the relation $\log _{10} c_{200}=$ $0.905-0.101 \log _{10}\left(M_{200} / 10^{12} h^{-1} M_{\odot}\right)$ with an intrinsic scatter of $0.11(1 \sigma)$ [37]. For the latter, we impose the $c_{200}-M_{200}$ relation as a top-hat prior within the $3 \sigma$ range in our MCMC sampling, together with an additional constraint on $V_{\max }, 1 / \sqrt{2}<V_{\max } / V_{\mathrm{f}}<\sqrt{2}$. The resulting inferences (median and $1 \sigma$ error) are shown in the figure. The two results agree well with each other. It is remarkable that even with the stringent constraints on $V_{\max }$ and $r_{\max }$ (through the $c_{200}-M_{200}$ relation), the SIDM halo model is able to fit the diverse rotation curves, as illustrated in Figs. 1 and 2 (left panel). Indeed, with the concentrationmass relation, we find that the $\Upsilon_{\star \text {,disk }}$ distribution is peaked toward $0.5 M_{\odot} / L_{\odot}$ in the fits, as shown in Fig. 2 (middle panel).

To see the MOND acceleration scale emerging from the hierarchical structure formation model, we parametrize a 
CDM halo with its gravitational acceleration at $r=0$ as $g_{\mathrm{NFW}}(0)=G M /\left.r^{2}\right|_{r \rightarrow 0} \approx 2 \pi G \rho_{s} r_{s} \approx 2 \pi V_{\max }^{2} /\left(1.26 r_{\max }\right)$, where $V_{\max } \approx \sqrt{0.58 G \rho_{s} r_{\max }^{2}}$ and $r_{\max } \approx 2.16 r_{s}$. Taking the mean cosmological $V_{\max }-r_{\max }$ relation, $r_{\max }=$ $27 \mathrm{kpc}\left(V_{\max } / 100 \mathrm{~km} / \mathrm{s}\right)^{1.4}$, we have $g_{\mathrm{NFW}}(0) \approx 1.0 \times$ $10^{-10} \mathrm{~m} / \mathrm{s}^{2}\left(V_{\max } / 240 \mathrm{~km} / \mathrm{s}\right)^{0.6}$, which is close to the MOND acceleration parameter $g_{*}$. This result is the underlying reason why the empirical MOND relation captures the overall stellar kinematics of spiral galaxies well. In the presence of dark matter self-interactions and baryons, the actual central acceleration deviates from $g_{\mathrm{NFW}}(0)$, but the general argument still holds. For example, we can characterize a halo with the acceleration at the scale radius $r_{s}$, where the impact of dark matter selfinteractions and the influence of baryons tend to be small, $g_{\mathrm{NFW}}\left(r_{s}\right) \approx 0.39 g_{\mathrm{NFW}}(0)$, slightly smaller than $g_{\mathrm{NFW}}$ at the center. The characteristic halo acceleration has a mild dependence on $V_{\max }$, ranging from 20 to $300 \mathrm{~km} / \mathrm{s}$ in the sample, and it also varies with the scatter in the cosmological relation. This variation is important, as shown in Fig. 3 (left panel). Since MOND does not have such flexibility, its overall fits are worse than the SIDM ones, as illustrated in Fig. 2 (right panel). We emphasize that $g_{\dagger}=$ $1.38 \times 10^{-10} \mathrm{~m} / \mathrm{s}^{2}$ inferred from our SIDM fits in Sec. III is an average quantity over the sample after fitting to Eq. (2), not a universal value for all the galaxies as in MOND (see also Ref. [59]).

The calculation of the acceleration due to dark matter toward the center is more subtle. Inside a constant density core, $g_{\text {SIDM }}(r) \propto r$, and we need to specify the radius where the acceleration is being computed. The half-light radius $\left(r_{1 / 2}\right)$, which encloses half the luminosity, is typically used to characterize the size of the stellar distribution, so it is a natural radius to compute the acceleration at $r_{1 / 2}$. On average, the stellar half-light radius is empirically observed to track the virial radius as $r_{1 / 2} \approx 0.015 r_{\text {vir }}$ [60], and we have $r_{1 / 2} \approx 1.7 R_{\mathrm{d}}$ for an exponential disk model. Without a significant contribution from baryons to the gravitational potential, SIDM predicts that $g_{\mathrm{SIDM}}\left(r_{1 / 2}\right)=10^{-11} \mathrm{~m} / \mathrm{s}^{2}$ $\left(V_{\max } / 100 \mathrm{~km} / \mathrm{s}\right)^{0.2}$ for the median halo concentration, and its dependence on the halo mass is extremely mild. When baryons contribute, $g_{\text {tot }}$ does not increase linearly with $g_{\text {bar }}$ since both the central SIDM density and the core radius depend on the gravitational potential contributed by the baryons. The net result is a strong correlation between $g_{\text {tot }}$ and $g_{\text {bar }}$, which is clearly evident in Fig. 2. The model predictions have a definite width in the $g_{\text {tot }}$ vs $g_{\text {bar }}$ plane, and we have shown clearly that this scatter is required to fully explain the diversity in the rotation curve data.

\section{CORRELATIONS BETWEEN THE TOTAL LUMINOUS AND DARK MATTER MASSES}

We have seen that the SIDM fits to the rotation curves require values for the halo concentration parameter that are completely in line with N-body simulations of structure formation using the cosmological parameters from the Planck experiment $[61,62]$. In addition, the stellar massto-light ratios are consistent with the results from stellar population models [31].

These results lead to a natural question: What is the predicted halo mass for a given stellar mass in the SIDM model? Since we assume the primordial matter power spectrum is unchanged from the CDM one for the scales we are interested in, there should be a relation consistent with the abundance matching results in the literature. In the middle panel of Fig. 3, we show the stellar mass vs halo mass relation derived using the mass-to-light ratios from controlled (circles) and MCMC (squares) samplings. The error bars on the MCMC points denote the $1 \sigma$ widths from the posteriors (16th and 84th percentiles). Our results are consistent with the overall trend in the relation from abundance matching (solid line) [58] (see Ref. [50] for the CDM case). We also note that there is a tendency for our data points to lie a bit to the left of the abundance matching line. The halo masses inferred from our fits seem to be systematically lower than those inferred in Ref. [58]. This difference could be due to different assumptions on the cosmological parameters in deriving the halo concentration-mass relation, or other differences in the analyses. A systematic investigation of this subject would be a fruitful avenue for future research. In addition, there are a few outliers on the left side of the black line in the low-mass regions, and many of them have low-quality observational data, as we will discuss later.

We have already alluded to the importance of the BTFR in our discussion of the RAR. Lelli et al. [54] selected 118 SPARC galaxies and found that their $V_{\mathrm{f}}-M_{\mathrm{bar}}$ inferences can be fitted with a simple relation: $\log \left(M_{\text {bar }}\right)=s \log \left(V_{\mathrm{f}}\right)+$ $\log (A)$, where $s=3.71 \pm 0.08$ and $\log (A)=2.27 \pm 0.18$ for $\Upsilon_{\star \text {,disk }}=\Upsilon_{\star \text {,bulge }}=0.5 M_{\odot} / L_{\odot}$. The right panel of Fig. 3 shows the $V_{\mathrm{f}}-M_{\text {bar }}$ inferences with the $\Upsilon_{\star \text {,disk }}$ and $\Upsilon_{\star \text {,bulge }}$ values from the controlled (circles) and MCMC (squares) fits. The error bars in $M_{\mathrm{bar}}$ on the MCMC points denote the $1 \sigma$ widths in the stellar massto-light ratios from the posteriors, and the errors in $V_{\mathrm{f}}$ are taken directly from the SPARC data set [18]. We also show the fit from Ref. [54] as the solid line of Fig. 3 (right panel). Note that this fit used 118 galaxies, and a few outliers at the low $V_{\mathrm{f}}$ end were not included. For comparison, we plot the 135 galaxies in our sample as triangles by fixing $\Upsilon_{\star \text {,disk }}=\Upsilon_{\star \text {,bulge }}=0.5 M_{\odot} / L_{\odot}$. We see that their distribution in the $V_{\mathrm{f}}-M_{\text {bar }}$ plane is almost identical to the one from our SIDM fits. This result is not surprising, as the $\Upsilon_{\star \text {,disk }}$ values inferred from the SIDM fits are peaked toward $0.5 M_{\odot} / L_{\odot}$ as shown in Fig. 2 (middle panel). Thus, we conclude that the SIDM fits also lead to a tight BTFR relation. For our fits, we find $s \approx 3.46(\mathrm{CS}), 3.27$ (MS), and $3.58\left(0.5 M_{\odot} / L_{\odot}\right)$, excluding six obvious outliers on the left side of the black line. Note that five of them, F 561-1, 
PGC 51017, UGC 04305, UGC06628, and UGC 09992, have either low-quality rotation curves or small inclination angles, and they may not be well suited for dynamical analysis. We have also checked that these galaxies are the outliers in the low-mass regions of the $M_{\text {star }}-M_{\text {halo }}$ relation, shown in Fig. 3 (middle panel).

We note that there is no evidence in the data for $s=4$ exactly, i.e., $M_{\mathrm{bar}} \propto V_{\mathrm{f}}^{4}$, which is the motivation for MOND, in either the constant $\Upsilon_{\star}$ fits or in the SIDM fits. We note that $V_{\mathrm{f}}$ may not be a good proxy for the asymptotic velocity of every galaxy in the sample, and systematic effects could lead to a shallower BTFR slope [63]. Many of the recent CDM simulations with efficient baryonic feedback seem to obtain something akin to the BTFR with $s \approx 3.6-3.8$ [64-67], but it is fair to say that this is still not well understood theoretically; in particular, the smallness of the scatter in the BTFR is equivalent to the one seen in the RAR [68]. We expect that there will be interplay between dark matter self-interactions and baryonic feedback in changing the halo potential. Understanding how the BTFR emerges in SIDM is fertile territory for research in galaxy formation.

\section{DISCUSSION AND CONCLUSIONS}

In this work, we have investigated SIDM as a solution to two puzzles that are present in galactic rotation curves: (1) the diversity of inner rotation curves in galaxies that have similar baryon content and similar flat circular velocities, and (2) the small scatter in the radial acceleration relation between the total gravitational acceleration and the one inferred from the baryonic mass content, i.e., uniformity.

We have fitted our SIDM halo model to the rotation curves of 135 SPARC galaxies and found that it reproduces the observed diversity in the inner regions. The distribution of the resulting 3.6- $\mu \mathrm{m}$ stellar disk mass-to-light ratios for the sample peaks at $\Upsilon_{\star \text {,disk }} \approx 0.5 M_{\odot} / L_{\odot}$, in good agreement with the stellar population models. Our fits lead to a radial acceleration relation described by the characteristic acceleration scale of about $10^{-10} \mathrm{~m} / \mathrm{s}^{2}$, with tight scatter of 0.10 dex. The host halos are fully consistent with the Planck cosmology. The inferred stellar mass-halo mass relation agrees with the result from the abundance matching method, and the fits also predict a tight BTFR. These results provide compelling arguments in favor of the idea that the inner halos of galaxies are kinematically thermalized due to dark matter self-interactions.

The SIDM model automatically inherits all of the successes of the CDM model on large scales, as the predictions are indistinguishable at distances larger than about $10 \%$ of the virial radius of galactic halos. The required cross section is similar to the proton-neutron elastic scattering cross section, and this similarity may be a strong hint that the dark matter sector replicates some elements of the standard model. The large cross section keeps the inner halo isothermal, and this makes the predictions for the central halo profile at later times insensitive to the star formation history, as confirmed in recent hydrodynamical $\mathrm{N}$-body simulations [35,69]. This result implies that a large variety of feedback models (e.g., Refs. [70-75]) can be compatible with the SIDM model we have discussed here. The predictions are quantitatively the same for $\sigma / \mathrm{m} \gtrsim 1 \mathrm{~cm}^{2} / \mathrm{g}$. This makes our results robust, but it is hard to precisely determine the cross section from kinematic data sets on galaxy scales [29].

There are a number of promising directions that can further test SIDM and explore galaxy formation and evolution in this framework. Here, we highlight a few of them. SIDM simulations predict a correlation between the half-light radius of the stars and the dark matter core size in dwarf and low-surface-brightness galaxies [27], which should be further explored and may provide an observational test of SIDM. Similarly, the ultradiffuse galaxies in the clusters could be a test laboratory [76]. A related issue is the origin of the large spread in the surface brightness of galaxies, which remains poorly understood. Interestingly, hydrodynamical simulations of galaxy clusters show that the stellar density profiles in SIDM are more diverse than in their CDM counterparts [35]. Is this a more general feature in SIDM due to the dynamical interplay between core formation and feedback? How does this interplay impact the emergence of the BTFR? Finally, at the lowest mass end, the dwarf spheroidal galaxies, including the so-called ultrafaint dwarfs, in the local group could provide a key test of SIDM (see Refs. [77,78]). Dedicated SIDM simulations with the baryons will be required to explore these exciting topics.

The predictive power of the SIDM model, the clear connection to cosmology, and its rich implications for other astrophysical observations and particle physics phenomenology [79], all taken together, make a clear case that it should be treated on the same footing as the CDM model. The economical explanation, with the addition of just one parameter, for the diverse rotation curves across the entire range of observed galaxies argues in favor of the idea that the dark matter particles have a large affinity for the selfinteractions.

\section{ACKNOWLEDGMENTS}

We thank the authors of Refs. [30,40,45] for the simulation data, which are used for comparison with the analytical model, shown in the Supplemental Material [32]. This work was supported by the National Science Foundation Grant No. PHY-1620638 (M. K.), the U.S. Department of Energy under Grant No. DE-SC0008541 (H. B. Y.), the Hellman Fellows Fund (H. B. Y.), UCR Academic Senate Regents Faculty Development Award (H. B. Y.), and the National Science Foundation under Grant No. NSF PHY-1748958 as part of the KITP "The Small-Scale Structure of Cold (?) Dark Matter" and "High 
Energy Physics at the Sensitivity Frontier" workshops (M. K. and H. B. Y.). H. B. Y. also acknowledges TsungDao Lee Institute, Shanghai, for its hospitality during the completion of this work.

\section{APPENDIX: METHODS}

We provide a detailed description of the analytical model developed previously $[26,29]$ and the fitting procedure. We divide the halo into an inner and an outer region [40], with the aim that the outer halo is not significantly changed by the self-scattering process. In the inner region, dark matter self-interactions thermalize the halo in the presence of the baryonic potential, and we model the dark matter distribution using the isothermal density profile, $\rho_{\text {iso }} \propto \exp \left(-\Phi_{\text {tot }}(R, z) / \sigma_{\mathrm{v} 0}^{2}\right)$. Poisson's equation relates $\Phi_{\text {tot }}$ to the dark matter and baryon profiles as

$$
\nabla^{2} \Phi_{\text {tot }}(R, z)=4 \pi G\left[\rho_{\text {iso }}(R, z)+\rho_{\mathrm{b}}(R, z)\right] .
$$

For the outer halo, where the self-scattering effect becomes negligible, we model the dark matter distribution with a NFW profile $\rho_{\mathrm{NFW}}(r)=\rho_{s} r_{s}^{3} / r\left(r+r_{s}\right)^{2}$. To construct the full SIDM halo profile, we define a radius $r_{1}$, where dark matter particles have one interaction on average over the age of the galaxy. We join the spherically averaged isothermal $\left(\rho_{\text {iso }}\right)$ and spherical NFW ( $\left.\rho_{\mathrm{NFW}}\right)$ profiles at $r=$ $r_{1}$ such that the mass and density are continuous at $r_{1}$. Thus, the isothermal parameters $\left(\rho_{0}, \sigma_{\mathrm{v} 0}\right)$ directly map onto the NFW parameters $\left(r_{s}, \rho_{s}\right)$ or $\left(r_{\max }, V_{\max }\right)$.

The value of $r_{1}$ is determined by the following condition,

$$
\left\langle\sigma v_{\text {rel }}\right\rangle \rho_{\mathrm{NFW}}\left(r_{1}\right) t_{\mathrm{age}} / m=N_{\mathrm{sc}},
$$

where $\sigma$ is the self-scattering cross section, $m$ is the dark matter particle mass, $v_{\text {rel }}$ is the dark matter relative velocity in the halo, $\langle\ldots\rangle$ denotes averaging over the Maxwellian velocity distribution, $t_{\text {age }}$ is the age of the galaxy, and $N_{\mathrm{sc}}$ is a factor of order unity, to be determined by calibrating to simulations. In this work, we have set $t_{\text {age }}=10 \mathrm{Gyr}$ and $N_{\text {sc }}=1$, which reproduce simulation results well; see the Supplemental Material [32]. In principle, we should use different ages for each galaxy, say, between 10 Gyr and 13 Gyr. However, our model can only constrain the combination of the cross section and the age. More importantly, we have set $\sigma / \mathrm{m}$ to a large enough value that the SIDM density profile is insensitive to small changes in the cross section. We assume that this cross section is a constant over the SPARC sample, so $\left\langle\sigma v_{\text {rel }}\right\rangle=\sigma(4 / \sqrt{\pi}) \sigma_{\mathrm{v} 0}$. In this work, we present our results for $\sigma / \mathrm{m}=3 \mathrm{~cm}^{2} / \mathrm{g}$ on galaxy scales, which can be naturally realized in particle physics models of SIDM [80-98].

We take two independent but complementary approaches. In the first one, we assume a thin-disk profile for the stellar disk in solving Eq. (A1), $\rho_{\mathrm{b}}(R, z)=\Sigma_{0} \exp \left(-R / R_{\mathrm{d}}\right) \delta(z)$, where $\Sigma_{0}$ is the central surface density and $R_{\mathrm{d}}$ is the scale radius. For each galaxy, we reconstruct the $\Sigma_{0}$ and $R_{\mathrm{d}}$ values by fitting the profile to the disk contribution of the rotation curve as in Ref. [29]. We neglect the baryonic influence on the SIDM halo from the gas and bulge potentials, but we include all the mass components in modeling the total circular velocity. This method is a reasonable approximation for the following reasons: (1) The gas is less centrally concentrated, so its impact on the SIDM density profile is smaller; (2) the bulge (when presents) mainly affects the innermost region, while the disk contributes in this region as well as at farther radii. Reference [29] solved Eq. (A1) with the thin-disk approximation and created numerical templates for the isothermal density profile on the grid of $a \equiv$ $8 \pi G \rho_{0} R_{\mathrm{d}}^{2} /\left(2 \sigma_{\mathrm{v} 0}^{2}\right)$ and $b \equiv 8 \pi G \Sigma_{0} R_{\mathrm{d}} /\left(2 \sigma_{\mathrm{v} 0}^{2}\right)$. When the stellar profile is known, the parameters $a$ and $b$ give the central density and dispersion of the isothermal dark matter halo, which completely specify the inner density profile. We interpolate the templates to generate rotation curves for any set of $\left(\rho_{0}, \sigma_{\mathrm{v} 0}, \Sigma_{0}, R_{\mathrm{d}}\right)$. The fixed value of the cross section allows us to match this density profile to the outer spherically symmetric NFW density profile. Since $r_{1} \sim r_{s} \gg R_{\mathrm{d}}$ in our fits, the influence of the baryons on the SIDM halo shape becomes negligible at $r_{1}$, and the SIDM halo recovers spherical symmetry accordingly; see the Supplemental Material [32].

In fitting to the SPARC sample with the templates, we take a controlled sampling approach. For a given galaxy, we start with the mean $r_{\max }-V_{\max }$ relation from cosmological $\Lambda$ CDM simulations [37] and a NFW profile that matches the flat part of the rotation curve. Then, we choose an appropriate $\Upsilon_{\star \text {,disk }}\left(\Upsilon_{\star \text {, bulge }}\right)$ value to reproduce the inner rotation curve. We calculate a $\chi^{2} /$ d.o.f. value for each fit and iterate this process manually by adjusting the parameters until a good fit is achieved. For most galaxies, the very first step provides decent fits, showcasing the simplicity of the model and its ability to fit the observed data simultaneously. For each galaxy, we demand the $\left(r_{\max }, V_{\max }\right)$ values to be within a band of about $2 \sigma$. In this way, we have good control over the halo parameters in the fits. The goal is to see to what degree the galaxy halos of the SPARC sample are consistent with predictions of the hierarchical structure formation scenario and to what degree the extracted $\Upsilon_{\star}$ values are consistent with stellar population synthesis model results [31].

In our second approach, we perform a MCMC sampling of the SIDM model parameter space. Since it is computationally expensive to use the templates, we use a spherical approximation to model the baryon distribution [25,28]. We create a spherical baryonic mass profile from the stellar and gas masses, such that the baryonic mass within a sphere of radius $r$ is $M_{\mathrm{b}}(r)=\left(V_{\text {disk }}^{2}+V_{\text {bulge }}^{2}+V_{\text {gas }}^{2}\right) r / G$, where $V_{\text {disk }}$ is the contribution to the rotation curve from the disk at radius $r$ and similarly for the bulge and gas. Below the smallest radii at which the baryonic contribution is 
tabulated in the SPARC database, we assume that the density in baryons is constant. Outside the last tabulated radii, we assume that the mass is constant. We have tested some galaxies where we change the constant central density to a smoothly matched Hernquist sphere [25] and found no difference.

We solve Eq. (A1) in the spherical limit by taking $r=$ $\sqrt{R^{2}+z^{2}}$ using the python module scipy.integrate.odeint, which uses LSODA from the FORTRAN library ODEPACK. We compute $\rho_{\text {iso }}(r)$ starting at a small radius $(10 \%$ of the innermost data point) assuming a core, i.e., $d \rho_{\text {iso }} / d r=0$, and integrate the equation to larger radii using the default settings in scipy.integrate.odeint. We compare the isothermal halos from this spherical approximation to those from the axisymmetric case (templates) and find agreement within 10\%-20\%. Thus, while we expect some variance in the inferred parameters between the two methods, the overall features should be very similar. This expectation is borne out by our final fits.

We match the isothermal density profile $\rho_{\text {iso }}$, parametrized by $\left(\rho_{0}, \sigma_{\mathrm{v} 0}\right)$, to the NFW density profile at $r_{1}$, and this determines $\left(V_{\max }, r_{\max }\right)$. Thus, the spherical model has four parameters, two for the entire halo and two for the mass-to-light ratios: $\left(\rho_{0}, \sigma_{\mathrm{v} 0}, \Upsilon_{\star \text {,disk }}, \Upsilon_{\star \text {,bulge }}\right)$. We use the emcee implementation of the affine invariant MCMC ensemble sampler [99] to infer the posteriors of these four model parameters. To streamline the calculation of $r_{1}$ at each point in parameter space for matching onto the outer NFW radius, we use the rate of scatterings, $\Gamma_{0}=\rho_{0}(\sigma / \mathrm{m})(4 / \sqrt{\pi}) \sigma_{\mathrm{v} 0}$, within the isothermal core as the MCMC parameter in lieu of the core density $\rho_{0}$.

The prior distributions used for the halo parameters and the mass-to-light ratios in the MCMC scan are as follows:

(i) $\Gamma_{0}$ : Uniform prior on $\log _{10} \Gamma_{0}$ in the range of $2<\Gamma_{0} \times 10 \mathrm{Gyr}<10^{5}$.

(ii) $\sigma_{0}$ : Uniform prior on $\log _{10} \sigma_{0}$ in the range of $2<\sigma_{0} /(\mathrm{km} / \mathrm{s})<500$.

(iii) $\Upsilon_{\star}$ : Uniform prior on both $\Upsilon_{\star \text {,disk }}$ and $\Upsilon_{\star \text {,bulge }}$ in the range of $0.1<\Upsilon_{\star} /\left(M_{\odot} / L_{\odot}\right)<10$. The parameter $\Upsilon_{\star \text { bulge }}$ is only included for galaxies whose surface brightness profiles have a stellar bulge decomposition provided in the SPARC data set. All galaxies have $\Upsilon_{\star \text {,disk }}$ as a parameter describing their stellar disk.

We have also added a term to the $\chi^{2}$ that imposes the $c_{200}-$ $M_{200}$ relation. This term has a mean value for $\log _{10} c_{200}$ given by the relation $0.905-0.101 \log _{10}\left(M_{200} / 10^{12} h^{-1} M_{\odot}\right)$ [37], with an error of 0.33 dex (top hat).

Additionally, we also impose two regularization priors.

(i) We add $5 \%$ of $V_{\mathrm{f}}$ in quadrature for calculating the likelihood function. This allows the code to disregard the points deep within the central regions and those with tiny errors so that they do not skew the fits. We have checked that it does not change the inference of cores or cusps. We do not include this regularization error when quoting $\chi^{2}$ values.

(ii) We impose a uniform regulation prior on $V_{\max }$ : $1 / \sqrt{2}<V_{\max } / V_{\mathrm{f}}<\sqrt{2}$. For most of the galaxies $(\sim 80 \%)$, our MCMC program can find physical fits without this prior. However, in some cases, the MCMC sampler tends to pick up fits that are not consistent with expectations based on the standard galaxy formation model; typically, the dark matter density is unreasonably low in the central regions. This result is due to the lack of an extended rotation curve to fully constrain the halo parameters. The additional regularization prior fixes this issue. We have also checked that the results are similar if we consider a more generous range $1 / 2<V_{\max } / V_{\mathrm{f}}<2$ (see the Supplemental Material [32]).

[1] R. A. Flores and J. R. Primack, Observational and Theoretical Constraints on Singular Dark Matter Halos, Astrophys. J. 427, L1 (1994).

[2] B. Moore, Evidence Against Dissipationless Dark Matter from Observations of Galaxy Haloes, Nature (London) 370, 629 (1994).

[3] A. Burkert, The Structure of Dark Matter Halos in Dwarf Galaxies, IAU Symp. 171, 175 (1996); Astrophys. J. 447, L25 (1995).

[4] M. Persic, P. Salucci, and F. Stel, The Universal Rotation Curve of Spiral Galaxies: 1. The Dark Matter Connection, Mon. Not. R. Astron. Soc. 281, 27 (1996).

[5] W. J. G. de Blok, S. S. McGaugh, A. Bosma, and V.C. Rubin, Mass Density Profiles of LSB Galaxies, Astrophys. J. 552, L23 (2001).

[6] G. Gentile, P. Salucci, U. Klein, D. Vergani, and P. Kalberla, The Cored Distribution of Dark Matter in Spiral Galaxies, Mon. Not. R. Astron. Soc. 351, 903 (2004).

[7] R. Kuzio de Naray, S. S. McGaugh, and W. de Blok, Mass Models for Low Surface Brightness Galaxies with High Resolution Optical Velocity Fields, Astrophys. J. 676, 920 (2008).

[8] W. J. G. de Blok, F. Walter, E. Brinks, C. Trachternach, S.-H. Oh, and R. C. Kennicutt, Jr., High-Resolution Rotation Curves and Galaxy Mass Models from THINGS, Astron. J. 136, 2648 (2008).

[9] S.-H. Oh, W. de Blok, E. Brinks, F. Walter, and R. C. Kennicutt, Dark and Luminous Matter in THINGS Dwarf Galaxies, Astron. J. 141, 193 (2011).

[10] S.-H. Oh et al., High-Resolution Mass Models of Dwarf Galaxies from LITTLE THINGS, Astron. J. 149, 180 (2015).

[11] R. Kuzio de Naray, G. D. Martinez, J. S. Bullock, and M. Kaplinghat, The Case Against Warm or Self-Interacting Dark Matter as Explanations for Cores in Low Surface Brightness Galaxies, Astrophys. J. 710, L161 (2010).

[12] K. A. Oman et al., The Unexpected Diversity of Dwarf Galaxy Rotation Curves, Mon. Not. R. Astron. Soc. 452, 3650 (2015). 
[13] J. S. Bullock and M. Boylan-Kolchin, Small-Scale Challenges to the $\Lambda \mathrm{CDM}$ Paradigm, Annu. Rev. Astron. Astrophys. 55, 343 (2017).

[14] M. Milgrom, A Modification of the Newtonian Dynamics as a Possible Alternative to the Hidden Mass Hypothesis, Astrophys. J. 270, 365 (1983).

[15] M. Milgrom, A Modification of the Newtonian Dynamics: Implications for Galaxies, Astrophys. J. 270, 371 (1983).

[16] B. Famaey and S. McGaugh, Modified Newtonian Dynamics (MOND): Observational Phenomenology and Relativistic Extensions, Living Rev. Relativity 15, 10 (2012).

[17] S. McGaugh, F. Lelli, and J. Schombert, Radial Acceleration Relation in Rotationally Supported Galaxies, Phys. Rev. Lett. 117, 201101 (2016).

[18] F. Lelli, S. S. McGaugh, and J. M. Schombert, SPARC: Mass Models for 175 Disk Galaxies with Spitzer Photometry and Accurate Rotation Curves, Astron. J. 152, 157 (2016).

[19] P. Li, F. Lelli, S. McGaugh, and J. Schormbert, Fitting the Radial Acceleration Relation to Individual SPARC Galaxies, Astron. Astrophys. 615, A3 (2018).

[20] F. C. van den Bosch and J. J. Dalcanton, Semi-analytical Models for the Formation of Disk Galaxies. 2. Dark Matter Versus Modified Newtonian Dynamics, Astrophys. J. 534, 146 (2000).

[21] M. Kaplinghat and M. S. Turner, How Cold Dark Matter Theory Explains Milgrom's Law, Astrophys. J. 569, L19 (2002).

[22] B. W. Keller and J. W. Wadsley, $\Lambda$ CDM is Consistent with SPARC Radial Acceleration Relation, Astrophys. J. 835, L17 (2017).

[23] A. D. Ludlow et al., Mass-Discrepancy Acceleration Relation: A Natural Outcome of Galaxy Formation in Cold Dark Matter Halos, Phys. Rev. Lett. 118, 161103 (2017).

[24] J. F. Navarro, A. Bentez-Llambay, A. Fattahi, C. S. Frenk, A. D. Ludlow, K. A. Oman, M. Schaller, and T. Theuns, The Origin of the Mass Discrepancy-Acceleration Relation in $\Lambda$ CDM, Mon. Not. R. Astron. Soc. 471, 1841 (2017).

[25] M. Kaplinghat, R. E. Keeley, T. Linden, and H.-B. Yu, Tying Dark Matter to Baryons with Self-interactions, Phys. Rev. Lett. 113, 021302 (2014).

[26] M. Kaplinghat, S. Tulin, and H.-B. Yu, Dark Matter Halos as Particle Colliders: Unified Solution to Small-Scale Structure Puzzles from Dwarfs to Clusters, Phys. Rev. Lett. 116, 041302 (2016).

[27] M. Vogelsberger, J. Zavala, C. Simpson, and A. Jenkins, Dwarf Galaxies in CDM and SIDM with Baryons: Observational Probes of the Nature of Dark Matter, Mon. Not. Roy. Astron. Soc. 444, 3684 (2014).

[28] O. D. Elbert, J. S. Bullock, M. Kaplinghat, S. GarrisonKimmel, A. S. Graus, and M. Rocha, A Testable Conspiracy: Simulating Baryonic Effects on Self-Interacting Dark Matter Halos, Astrophys. J. 853, 109 (2018).

[29] A. Kamada, M. Kaplinghat, A. B. Pace, and H.-B. Yu, How the Self-Interacting Dark Matter Model Explains the Diverse Galactic Rotation Curves, Phys. Rev. Lett. 119, 111102 (2017).

[30] P. Creasey, O. Sameie, L. V. Sales, H.-B. Yu, M. Vogelsberger, and J. Zavala, Spreading out and Staying Sharp-Creating Diverse Rotation Curves via Baryonic and
Self-Interaction Effects, Mon. Not. R. Astron. Soc. 468, 2283 (2017).

[31] J. M. Schombert and S. McGaugh, Stellar Populations and the Star Formation Histories of LSB Galaxies: IV Spitzer Surface Photometry of LSB Galaxies, Pub. Astron. Soc. Aust. 31, 11 (2014).

[32] See Supplemental Material at http://link.aps.org/ supplemental/10.1103/PhysRevX.9.031020 for additional information.

[33] J. F. Navarro, C. S. Frenk, and S. D. M. White, The Structure of Cold Dark Matter Halos, Astrophys. J. 462, 563 (1996).

[34] J. F. Navarro, C. S. Frenk, and S. D. White, A Universal Density Profile from Hierarchical Clustering, Astrophys. J. 490, 493 (1997).

[35] A. Robertson, R. Massey, V. Eke, S. Tulin, H.-B. Yu, Y. Bah, D. J. Barnes, C. D. Vecchia, and S. T. Kay, et al., The Diverse Density Profiles of Galaxy Clusters with Self-Interacting Dark Matter Plus Baryons, Mon. Not. R. Astron. Soc. 476, L20 (2018)..

[36] O. Sameie, P. Creasey, H.-B. Yu, L. V. Sales, M. Vogelsberger, and J. Zavala, The Impact of Baryonic Disks on the Shapes and Profiles of Self-Interacting Dark Matter Halos, Mon. Not. R. Astron. Soc. 479, 359 (2018)..

[37] A. A. Dutton and A. V. Macciò, Cold Dark Matter Haloes in the Planck Era: Evolution of Structural Parameters for Einasto and NFW Profiles, Mon. Not. R. Astron. Soc. 441, 3359 (2014).

[38] D. N. Spergel and P. J. Steinhardt, Observational Evidence for Self-Interacting Cold Dark Matter, Phys. Rev. Lett. 84, 3760 (2000).

[39] R. Dave, D. N. Spergel, P. J. Steinhardt, and B. D. Wandelt, Halo Properties in Cosmological Simulations of Self-Interacting Cold Dark Matter, Astrophys. J. 547, 574 (2001).

[40] M. Rocha, A. H. G. Peter, J. S. Bullock, M. Kaplinghat, S. Garrison-Kimmel, J. Onorbe, and L. A. Moustakas, Cosmological Simulations with Self-Interacting Dark Matter I: Constant Density Cores and Substructure, Mon. Not. R. Astron. Soc. 430, 81 (2013).

[41] A. H. G. Peter, M. Rocha, J. S. Bullock, and M. Kaplinghat, Cosmological Simulations with Self-Interacting Dark Matter II: Halo Shapes vs. Observations, Mon. Not. R. Astron. Soc. 430, 105 (2013).

[42] M. Vogelsberger, J. Zavala, and A. Loeb, Subhaloes in SelfInteracting Galactic Dark Matter Haloes, Mon. Not. R. Astron. Soc. 423, 3740 (2012).

[43] J. Zavala, M. Vogelsberger, and M. G. Walker, Constraining Self-Interacting Dark Matter with the Milky Way's Dwarf Spheroidals, Mon. Not. R. Astron. Soc. 431, L20 (2013).

[44] M. Vogelsberger, J. Zavala, F.-Y. Cyr-Racine, C. Pfrommer, T. Bringmann, and K. Sigurdson, ETHOS-An Effective Theory of Structure Formation: Dark Matter Physics as a Possible Explanation of the Small-Scale CDM Problems, Mon. Not. R. Astron. Soc. 460, 1399 (2016).

[45] O. D. Elbert, J. S. Bullock, S. Garrison-Kimmel, M. Rocha, J. Oorbe, and A. H. G. Peter, Core Formation in Dwarf Haloes with Self-Interacting Dark Matter: No Fine-Tuning Necessary, Mon. Not. R. Astron. Soc. 453, 29 (2015).

[46] A. Sokolenko, K. Bondarenko, T. Brinckmann, J. Zavala, M. Vogelsberger, T. Bringmann, and A. Boyarsky, Towards 
an Improved Model of Self-Interacting Dark Matter Haloes, arXiv:1806.11539.

[47] C. Firmani, E. D’Onghia, V. Avila-Reese, G. Chincarini, and X. Hernandez, Evidence of Self-Interacting Cold Dark Matter from Galactic to Galaxy Cluster Scales, Mon. Not. R. Astron. Soc. 315, L29 (2000).

[48] N. Yoshida, V. Springel, S. D. M. White, and G. Tormen, Weakly Self-Interacting Dark Matter and the Structure of Dark Halos, Astrophys. J. 544, L87 (2000).

[49] M. T. Frandsen and J. Petersen, Investigating Dark Matter and MOND Models with Galactic Rotation Curve Data, arXiv:1805.10706.

[50] H. Desmond, A Statistical Investigation of the Mass Discrepancy-Acceleration Relation, Mon. Not. R. Astron. Soc. 464, 4160 (2017).

[51] S. S. McGaugh, J. M. Schombert, G. D. Bothun, and W. J. G. de Blok, The Baryonic Tully-Fisher Relation, Astrophys. J. 533, L99 (2000).

[52] J. D. Bradford, M. C. Geha, and F. C. van den Bosch, A Slippery Slope: Systematic Uncertainties in the Line Width Baryonic Tully-Fisher Relation, Astrophys. J. 832, 11 (2016).

[53] A. A. Ponomareva, M. A. W. Verheijen, E. Papastergis, A. Bosma, and R. F. Peletier, From Light to Baryonic Mass: The Effect of the Stellar Mass-to-Light Ratio on the Baryonic Tully-Fisher Relation, Mon. Not. R. Astron. Soc. 474, 4366 (2018).

[54] F. Lelli, S. S. McGaugh, and J. M. Schombert, The Small Scatter of the Baryonic Tully-Fisher Relation, Astrophys. J. 816, L14 (2016).

[55] F. Prada, A. A. Klypin, A. J. Cuesta, J. E. Betancort-Rijo, and J. Primack, Halo Concentrations in the Standard LCDM Cosmology, Mon. Not. R. Astron. Soc. 423, 3018 (2012).

[56] A. D. Ludlow, J. F. Navarro, R. E. Angulo, M. Boylan-Kolchin, V. Springel, C. Frenk, and S. D. M. White, The Mass-Concentration-Redshift Relation of Cold Dark Matter Haloes, Mon. Not. R. Astron. Soc. 441, 378 (2014).

[57] H. Katz, F. Lelli, S. S. McGaugh, A. Di Cintio, C. B. Brook, and J.M. Schombert, Testing Feedback-Modified Dark Matter Haloes with Galaxy Rotation Curves: Estimation of Halo Parameters and Consistency with CDM Scaling Relations, Mon. Not. R. Astron. Soc. 466, 1648 (2017).

[58] P. S. Behroozi, R. H. Wechsler, and C. Conroy, The Average Star Formation Histories of Galaxies in Dark Matter Halos from $z=0-8$, Astrophys. J. 770, 57 (2013).

[59] D. C. Rodrigues, V. Marra, A. del Popolo, and Z. Davari, Absence of a Fundamental Acceleration Scale in Galaxies, Nat. Astron. 2, 668 (2018).

[60] A. V. Kravtsov, The Size-Virial Radius Relation of Galaxies, Astrophys. J. 764, L31 (2013).

[61] P. A. R. Ade et al. (Planck Collaboration), Planck 2013 Results. XVI. Cosmological Parameters, Astron. Astrophys. 571, A16 (2014).

[62] P. A. R. Ade et al. (Planck Collaboration), Planck 2015 Results. XIII. Cosmological Parameters, Astron. Astrophys. 594, A13 (2016).

[63] P. Li, F. Lelli, S. S. McGaugh, N. Starkman, and J. M. Schombert, A Constant Characteristic Volume Density of
Dark Matter Haloes from SPARC Rotation Curve Fits, Mon. Not. R. Astron. Soc. 482, 5106 (2019).

[64] A. A. Dutton, The Baryonic Tully-Fisher Relation and Galactic Outflows, Mon. Not. R. Astron. Soc. 424, 3123 (2012).

[65] T. K. Chan, D. Kere, J. Oorbe, P. F. Hopkins, A. L. Muratov, C. A. Faucher-Gigure, and E. Quataert, The Impact of Baryonic Physics on the Structure of Dark Matter Haloes: The View from the FIRE Cosmological Simulations, Mon. Not. R. Astron. Soc. 454, 2981 (2015).

[66] L. V. Sales et al., The Low-Mass End of the Baryonic Tully-Fisher Relation, Mon. Not. R. Astron. Soc. 464, 2419 (2017).

[67] A. A. Dutton, A. Obreja, L. Wang, T. A. Gutcke, T. Buck, S. M. Udrescu, J. Frings, G. S. Stinson, X. Kang, and A. V. Macci, NIHAO XII: Galactic Uniformity in a $\Lambda \mathrm{CDM}$ Universe, Mon. Not. R. Astron. Soc. 467, 4937 (2017).

[68] C. Wheeler, P.F. Hopkins, and O. Doré, The Radial Acceleration Relation Is a Natural Consequence of the Baryonic Tully-Fisher Relation, arXiv:1803.01849.

[69] V. H. Robles, J. S. Bullock, O. D. Elbert, A. Fitts, A. González-Samaniego, M. Boylan-Kolchin, P. F. Hopkins, C.-A. Faucher-Giguère, D. Kereš, and C. C. Hayward, SIDM on FIRE: Hydrodynamical Self-Interacting Dark Matter Simulations of Low-Mass Dwarf Galaxies, Mon. Not. R. Astron. Soc. 472, 2945 (2017).

[70] F. Governato, A. Zolotov, A. Pontzen, C. Christensen, S. H. Oh, A. M. Brooks, T. Quinn, S. Shen, and J. Wadsley, Cuspy No More: How Outflows Affect the Central Dark Matter and Baryon Distribution in Lambda CDM Galaxies, Mon. Not. R. Astron. Soc. 422, 1231 (2012).

[71] P. F. Hopkins, D. Keres, J. Onorbe, C.-A. Faucher-Giguere, E. Quataert, N. Murray, and J. S. Bullock, Galaxies on FIRE (Feedback In Realistic Environments): Stellar Feedback Explains Cosmologically Inefficient Star Formation, Mon. Not. R. Astron. Soc. 445, 581 (2014).

[72] J. Oorbe, M. Boylan-Kolchin, J. S. Bullock, P. F. Hopkins, D. Kers, C.-A. Faucher-Gigure, E. Quataert, and N. Murray, Forged in FIRE: Cusps, Cores, and Baryons in Low-Mass Dwarf Galaxies, Mon. Not. R. Astron. Soc. 454, 2092 (2015).

[73] L. Wang, A. A. Dutton, G. S. Stinson, A. V. Macci, C. Penzo, X. Kang, B. W. Keller, and J. Wadsley, NIHAO Project I. Reproducing the Inefficiency of Galaxy Formation across Cosmic Time with a Large Sample of Cosmological Hydrodynamical Simulations, Mon. Not. R. Astron. Soc. 454, 83 (2015).

[74] J. I. Read, G. Iorio, O. Agertz, and F. Fraternali, The Stellar Mass-Halo Mass Relation of Isolated Field Dwarfs: A Critical Test of $\Lambda \mathrm{CDM}$ at the Edge of Galaxy Formation, Mon. Not. R. Astron. Soc. 467, 2019 (2017).

[75] I. M. Santos-Santos, A. Di Cintio, C. B. Brook, A. Macciò, A. Dutton, and R. Domínguez-Tenreiro, NIHAO-XIV. Reproducing the Observed Diversity of Dwarf Galaxy Rotation Curve Shapes in $\Lambda \mathrm{CDM}$, Mon. Not. R. Astron. Soc. 473, 4392 (2018).

[76] T. Carleton, R. Errani, M. Cooper, M. Kaplinghat, and J. Peñarrubia, The Formation of Ultra Diffuse Galaxies in Cored Dark Matter Halos through Tidal Stripping and Heating, arXiv:1805.06896. 
[77] M. Valli and H.-B. Yu, Dark Matter Self-Interactions from the Internal Dynamics of Dwarf Spheroidals, Nat. Astron.2, 907 (2018).

[78] J. I. Read, M. G. Walker, and P. Steger, The Case for a Cold Dark Matter Cusp in Draco, arXiv:1805.06934.

[79] S. Tulin and H.-B. Yu, Dark Matter Self-Interactions and Small Scale Structure, Phys. Rep. 730, 1 (2018).

[80] J. L. Feng, M. Kaplinghat, H. Tu, and H.-B. Yu, Hidden Charged Dark Matter, J. Cosmol. Astropart. Phys. 07 (2009) 004.

[81] J. L. Feng, M. Kaplinghat, and H.-B. Yu, Halo Shape and Relic Density Exclusions of Sommerfeld-Enhanced Dark Matter Explanations of Cosmic Ray Excesses, Phys. Rev. Lett. 104, 151301 (2010).

[82] M. R. Buckley and P. J. Fox, Dark Matter Self-Interactions and Light Force Carriers, Phys. Rev. D 81, 083522 (2010).

[83] A. Loeb and N. Weiner, Cores in Dwarf Galaxies from Dark Matter with a Yukawa Potential, Phys. Rev. Lett. 106, 171302 (2011).

[84] M. T. Frandsen and S. Sarkar, Asymmetric Dark Matter and the Sun, Phys. Rev. Lett. 105, 011301 (2010).

[85] M. T. Frandsen, S. Sarkar, and K. Schmidt-Hoberg, Light Asymmetric Dark Matter from New Strong Dynamics, Phys. Rev. D 84, 051703(R) (2011).

[86] L. G. van den Aarssen, T. Bringmann, and C. Pfrommer, Is Dark Matter with Long-Range Interactions a Solution to All Small-Scale Problems of Lambda CDM Cosmology?, Phys. Rev. Lett. 109, 231301 (2012).

[87] J. M. Cline, Z. Liu, G. Moore, and W. Xue, Composite Strongly Interacting Dark Matter, Phys. Rev. D 90, 015023 (2014).

[88] F. Kahlhoefer, K. Schmidt-Hoberg, M. T. Frandsen, and S. Sarkar, Colliding Clusters and Dark Matter Self-Interactions, Mon. Not. R. Astron. Soc. 437, 2865 (2014).

[89] S. Tulin, H.-B. Yu, and K. M. Zurek, Beyond Collisionless Dark Matter: Particle Physics Dynamics for Dark Matter Halo Structure, Phys. Rev. D 87, 115007 (2013).
[90] K. Schutz and T. R. Slatyer, Self-Scattering for Dark Matter with an Excited State, J. Cosmol. Astropart. Phys. 01 (2015) 021.

[91] K. K. Boddy, J. L. Feng, M. Kaplinghat, and T. M. P. Tait, Self-Interacting Dark Matter from a Non-Abelian Hidden Sector, Phys. Rev. D 89, 115017 (2014).

[92] Y. Hochberg, E. Kuflik, T. Volansky, and J. G. Wacker, Mechanism for Thermal Relic Dark Matter of Strongly Interacting Massive Particles, Phys. Rev. Lett. 113, 171301 (2014).

[93] Y. Hochberg, E. Kuflik, H. Murayama, T. Volansky, and J. G. Wacker, Model for Thermal Relic Dark Matter of Strongly Interacting Massive Particles, Phys. Rev. Lett. 115, 021301 (2015).

[94] F.-Y. Cyr-Racine, K. Sigurdson, J. Zavala, T. Bringmann, M. Vogelsberger, and C. Pfrommer, ETHOS_An Effective Theory of Structure Formation: From Dark Particle Physics to the Matter Distribution of the Universe, Phys. Rev. D 93, 123527 (2016).

[95] M. Blennow, S. Clementz, and J. Herrero-Garcia, SelfInteracting Inelastic Dark Matter: A Viable Solution to the Small Scale Structure Problems, J. Cosmol. Astropart. Phys. 03 (2017) 048.

[96] K. K. Boddy, M. Kaplinghat, A. Kwa, and A. H. G. Peter, Hidden Sector Hydrogen as Dark Matter: SmallScale Structure Formation Predictions and the Importance of Hyperfine Interactions, Phys. Rev. D 94, 123017 (2016).

[97] A. Kamada, H. J. Kim, and H. Kim, Self-Heating of Strongly Interacting Massive Particles, Phys. Rev. D 98, 023509 (2018).

[98] E. Braaten, D. Kang, and R. Laha, Production of DarkMatter Bound States in the Early Universe by Three-Body Recombination, J. High Energy Phys. 11 (2018) 084.

[99] D. Foreman-Mackey, D. W. Hogg, D. Lang, and J. Goodman, emcee: The MCMC Hammer, Publ. Astron. Soc. Pac. 125, 306 (2013). 